\title{
Direct Hyperbilirubinemia as a Predictor of Mortality in Patients with Liver Cirrhosis
}

\author{
Chang Hun Lee and In Hee Kim \\ Department of Internal Medicine, Research Institute of Clinical Medicine of Jeonbuk National University-Biomedical Research Institute \\ of Jeonbuk National University Hospital, Jeonbuk National University Medical School, Jeonju, Korea
}

\author{
Corresponding Author \\ Chang Hun Lee \\ ORCID https://orcid.org/0000-0001-7244-8849 \\ E-mail chleegi@jbnu.ac.kr
}

\author{
See "Direct Bilirubin Is More Valuable than Total Bilirubin for Predicting Prognosis in Pa- \\ tients with Liver Cirrhosis" by Han Ah Lee, et al. on page 599, Vol. 15, No. 4, 2021
}

Liver cirrhosis (LC) is the 11th leading cause of death globally, and its prevalence has been increasing in recent decades. ${ }^{1}$ Because of the high fatality rate associated with LC, there have been many attempts to identify accurate prognostic models that will help make better clinical decisions, but these attempts have not led to satisfactory results. ${ }^{2,3}$ Histopathologic findings and hepatic venous pressure gradient may reflect disease severity, but their clinical use is limited due to their invasiveness. ${ }^{4}$ Widely used noninvasive parameters include the Child-Pugh score and Model for End-Stage Liver Disease (MELD) score-the MELD system is noted for its use in liver transplant allocation. ${ }^{5,6}$ These two models use serum bilirubin as a component of their prognostic scales. Most previous studies have used total bilirubin (TB), rather than direct bilirubin (DB), as an indicator of bilirubin metabolism. This paper presents a study that focuses on DB and evaluates its relevance in predicting poor outcomes.

Lee et al. ${ }^{7}$ compared the prognostic performance of DB and TB in patients with LC. They retrospectively assessed 983 patients who were admitted to the hospital during the study period; the patients were divided into two groups: a training set and a validation set. Lee et al. evaluated significance using the $\mathrm{C}$-statistics for survival and compared the performance of MELD score and DB-MELD score (MELD score determined using DB instead of TB). The results showed that the prognostic performance of $\mathrm{DB}$ was higher than that of TB, and DB-MELD score showed a higher area under the receiver operating characteristic curve than the MELD score. Moreover, Lee et al. reweighed the components with beta coefficients and developed a new prediction model: "Direct Bilirubin, INR, and Creatinine" (DiBIC) score. The DiBIC score showed high performance not only in the training set but also in the validation set in terms of 6-month mortality prediction.

Bilirubin, a breakdown product of heme-containing proteins, exists in the blood in two forms: conjugated (DB) and unconjugated (indirect bilirubin). In chronic liver diseases, pathologic elevation of conjugated bilirubin is predominant, and DB and TB levels usually rise together. Hepatic clearance of bilirubin occurs in a series of steps: (1) the uptake of unconjugated bilirubin by hepatocytes; (2) the conjugation of bilirubin; (3) the excretion of conjugated bilirubin into bile ducts; and (4) the reuptake of conjugate bilirubin by hepatocytes. Elevation of the unconjugated fraction may result from overproduction, reduced hepatic uptake, or defective bilirubin conjugation-all of which may be affected by portal flow distortion, portosystemic shunting, and splenomegaly in the cirrhotic liver. ${ }^{8}$ Elevation of conjugated bilirubin may be the result of impaired hepatic excretory ability, the rate-limiting step in bilirubin metabolism. A disrupted transport system of the bile into the biliary canaliculi, intrahepatic cholestasis, or backward leakage from damaged hepatocytes can contribute to direct hyperbilirubinemia. ${ }^{9}$ Deterioration of bile egress leads to the accumulation of potentially toxic compounds in the liver and circulation, resulting in liver damage. ${ }^{10}$ Lee et al. ${ }^{7}$ supported that a defect in hepatic clearance due to a damaged liver is responsible for poor prognosis in patients with LC. Further mechanistic studies elucidating bilirubin 
metabolism and the relationship between DB levels and prognosis are required.

The study by Lee et al. ${ }^{7}$ has several limitations. Firstly, this was a single-center retrospective study, though the study did have a large sample size. Secondly, the MELD model was not designed using DB and was originally created to predict 3-month mortality (this study analyzed 6-month mortality probability because the 3 -month death rate was very low). Moreover, they used the value of $\mathrm{DB}$ by adding 1 to DB-MELD because the value of DB was too low, which could skew the results. Thirdly, better prediction using DB may not only depend on the fraction of bilirubin, but it may also be influenced by the reweighting of components. Lastly, the newly developed prognostic model, DiBIC score, should be tested for external validity.

Precise assessment of prognosis may facilitate proper care of patients with a high risk of mortality. Lee et al. ${ }^{7}$ have raised important points for clinical application: they elucidated the superiority of $\mathrm{DB}$ over TB in terms of prognostic performance for LC, and they developed DB-based prediction models (DB-MELD and DiBIC) and reweighted the components of MELD. Further research on the mechanistic analysis of bilirubin and external validation of the newly developed model to supplement this study for patients with LC is warranted.

\section{CONFLICTS OF INTEREST}

No potential conflict of interest relevant to this article was reported.

\section{ORCID}

Chang Hun Lee

https://orcid.org/0000-0001-7244-8849

In Hee Kim

https://orcid.org/0000-0003-3863-7907

\section{REFERENCES}

1. Asrani SK, Devarbhavi H, Eaton J, Kamath PS. Burden of liver diseases in the world. J Hepatol 2019;70:151-171.

2. Durand F, Valla D. Assessment of prognosis of cirrhosis. Semin Liver Dis 2008;28:110-122.

3. Chen SH, Wan QS, Wang T, Zhang KH. Fluid biomarkers for predicting the prognosis of liver cirrhosis. Biomed Res Int 2020;2020:7170457.

4. Ripoll C, Groszmann R, Garcia-Tsao G, et al. Hepatic venous pressure gradient predicts clinical decompensation in patients with compensated cirrhosis. Gastroenterology 2007;133:481-488.

5. D’Amico G, Garcia-Tsao G, Pagliaro L. Natural history and prognostic indicators of survival in cirrhosis: a systematic review of 118 studies. J Hepatol 2006;44:217-231.

6. Kamath PS, Kim WR; Advanced Liver Disease Study Group. The model for end-stage liver disease (MELD). Hepatology 2007;45:797-805

7. Lee HA, Jung JY, Lee YS, et al. Direct bilirubin is more valuable than total bilirubin for predicting prognosis in patients with liver cirrhosis. Gut Liver 2121;15:599-605.

8. Ohkubo A. Bilirubin metabolism in liver cirrhosis. Nihon Rinsho 1994;52:138-144.

9. Aravinthan AD, Alexander GJ. Hepatocyte senescence explains conjugated bilirubinaemia in chronic liver failure. J Hepatol 2015;63:532-533.

10. López-Velázquez JA, Chávez-Tapia NC, Ponciano-Rodríguez $\mathrm{G}$, et al. Bilirubin alone as a biomarker for short-term mortality in acute-on-chronic liver failure: an important prognostic indicator. Ann Hepatol 2013;13:98-104. 